\title{
A novel prognostic model: which group of esophageal squamous cell carcinoma patients could benefit from adjuvant chemotherapy
}

\author{
Zihao Zhou $^{1 \#}$, Shujie Huang ${ }^{1,2 \#}$, Xiaosong Ben ${ }^{1}$, Weitao Zhuang ${ }^{1,2}$, Liangli Hong ${ }^{3}$, Zefeng Xie ${ }^{4}$, \\ Dongkun Zhang ${ }^{1}$, Liang Xie ${ }^{1}$, Haiyu Zhou ${ }^{1}$, Jiming Tang ${ }^{1}$, Gang Chen ${ }^{1}$, Hansheng Wu ${ }^{1,4}$, Guibin Qiao, \\ ${ }^{1}$ Department of Thoracic Surgery, Guangdong Provincial People's Hospital, Guangdong Academy of Medical Sciences, Guangzhou, China; ${ }^{2}$ Shantou \\ University Medical College, Shantou, China; ${ }^{3}$ Department of Pathology, The First Affiliated Hospital of Shantou University Medical College, \\ Shantou, China; ${ }^{4}$ Department of Thoracic Surgery, The First Affiliated Hospital of Shantou University Medical College, Shantou, China; ${ }^{5}$ The \\ Second School of Clinical Medicine, Southern Medical University, Guangzhou, China \\ Contributions: (I) Conception and design: Z Zhou, S Huang; (II) Administrative support: H Wu, G Qiao; (III) Provision of study materials or patients: \\ X Ben, D Zhang, L Xie, H Zhou, J Tang, G Chen, H Wu, G Qiao; (IV) Collection and assembly of data: Z Zhou, S Huang, W Zhuang; (V) Data \\ analysis and interpretation: Z Zhou, S Huang; (VI) Manuscript writing: All authors; (VII) Final approval of manuscript: All authors. \\ \#These authors contributed equally to this work. \\ Correspondence to: Guibin Qiao, MD, PhD. Department of Thoracic Surgery, Guangdong Provincial People's Hospital, Guangdong Academy \\ of Medical Sciences, Guangzhou 510080, China; The Second School of Clinical Medicine, Southern Medical University, Guangzhou, China. \\ Email: guibinqiao@126.com; Hansheng Wu. Department of Thoracic Surgery, Guangdong Provincial People’s Hospital, Guangdong Academy \\ of Medical Sciences, Guangzhou 510080, China; Department of Thoracic Surgery, The First Affiliated Hospital of Shantou University Medical \\ College, Shantou, China. Email: wu-han-sheng@163.com.
}

Background: This study aimed to establish a reliable model for predicting the overall survival (OS) of esophageal squamous cell carcinoma (ESCC) patients and identifying the potential beneficiaries of adjuvant chemotherapy after esophagectomy.

Methods: This retrospective study included 819 ESCC patients who underwent esophagectomy as the training cohort. We constructed a prognostic model named GTLN2. Both internal and external validation tests were performed. Potential beneficiaries were defined as ESCC patients who obtained a significantly longer OS after adjuvant chemotherapy. Propensity score matching (PSM) was utilized in the subgroup analysis to screen ESCC beneficiaries of adjuvant chemotherapy.

Results: We enrolled a total of 819 cT1b-3 patients in the training cohort. Multiple prognostic factors were associated with adjuvant chemotherapy. Using uni-/multivariate analysis, histological grade (G), tumor invasion depth $(\mathrm{T})$, regional lymph node metastasis $(\mathrm{N})$, and the number of cleared lymph nodes (NCLNs) were identified as independent prognostic factors. Then, we developed the GTLN2 model based on these predictors and validated it using internal calculations [the 1-, 3- and 5-year area under the curves (AUCs) were 0.692, 0.685 and 0.680, respectively; $\mathrm{P}<0.001$ ] and external cohorts (the 1-, 3-, and 5-year AUCs were 0.651, 0.619 and 0.650, respectively; $\mathrm{P}<0.001$ ). ESCC patients were categorized into high- and low-risk groups based on their assigned risk scores. After 1:1 patient pairing was performed by PSM in the high-risk group, better OS was noted in patients receiving adjuvant chemotherapy $(\mathrm{P}=0.024)$.

Conclusions: Differentiating high- and low-risk patient groups via a novel mathematical prediction model allows physicians to identify patients in need of adjuvant chemotherapy accurately.

Keywords: Esophageal squamous cell carcinoma (ESCC); adjuvant chemotherapy; prognostic model; overall survival (OS); real-world study

Submitted Dec 14, 2021. Accepted for publication Jan 20, 2022.

doi: $10.21037 / \mathrm{atm}-22-46$

View this article at: https://dx.doi.org/10.21037/atm-22-46 


\section{Introduction}

Esophageal cancer is a malignant disease and is a serious health problem. Approximately 450,000 patients are diagnosed with esophageal cancer each year worldwide (1). In China, esophageal squamous cell carcinoma (ESCC) is the primary histological type (2).

Surgery is the mainstay of treatment for medically fit cT1b-4a ESCC patients (3). Increasing the assessment of multidisciplinary approaches to improve the postoperative survival rate and quality of life of ESCC patients has reached a mature stage. Among the adopted therapeutic approaches, the effect of adjuvant chemotherapy on esophageal cancer has been heatedly debated. In a previous study, adjuvant chemotherapy was revealed to offer survival benefits in patients with ESCC (4). Similar findings were reported by Wong et al. (5). In another meta-analysis, adjuvant chemotherapy significantly improved both overall survival (OS) and disease-free survival (DFS) (6). Conversely, a multicenter retrospective study conducted by Pasquer et al. showed that adjuvant chemotherapy was not associated with improved survival after resection in ESCC patients (7). To date, no phase III randomized controlled trial has shown a survival benefit of adjuvant chemotherapy (8). The current National Comprehensive Cancer Network (NCCN) guidelines for esophageal cancer do not define the benefit of adjuvant chemotherapy. For resectable ESCC, adjuvant chemotherapy alone is recommended for late-stage disease, which requires palliative management.

Currently, no well-established prognostic models are available for predicting the survival of patients receiving adjuvant chemotherapy. For widely used prognostic models such as the tumor-nodal-metastasis (TNM) staging system, accumulating data have suggested that they cannot offer valid information for the selection of ESCC beneficiaries of adjuvant chemotherapy $(9,10)$. Duan et al. constructed a reliable nomogram on stratifying the prognosis of ESCC patients receiving adjuvant chemotherapy, the concordance index of which showed more superior predictive power than TNM staging system (11). However, the external validity the prognostic model remained to be confirmed. To date, no report has included an externally validated scoring system for screening ESCC patient groups.

Hence, this work developed and externally validated a prognostic scoring system integrated with independent clinicopathological factors. The prognostic model was named GTLN2 for the reason that four independent variables histological grade $(\mathrm{G})$, tumor invasion depth $(\mathrm{T})$, regional lymph node metastasis $(\mathrm{N})$, and the number of cleared lymph nodes (NCLNs) were integrated into the system. To predict survival differences and the beneficial effects of chemotherapeutics among distinctive patient groups, we stratified ESCC patients with different characteristics based on the assigned scores.

We present the following article in accordance with the STROBE reporting checklist (available at https://atm. amegroups.com/article/view/10.21037/atm-22-46/rc)

\section{Methods}

\section{Patient characteristics}

A total of 819 patients with ESCC who underwent esophagectomy at the Department of Thoracic Surgery, Guangdong Provincial People's Hospital between January 2009 and December 2019 were enrolled as the training cohort. In addition, the validation cohort consisted of 430 patients who underwent esophagectomy between January 2010 and November 2019 at the Department of Thoracic Surgery, The First Affiliated Hospital of Shantou University Medical College. Detailed clinicopathological characteristics of the study population were assessed. The inclusion criteria were as follows: (I) p T1b-3N0$3 \mathrm{M0}$ patients who underwent esophagectomy surgery; (II) pathologic confirmation of ESCC; (III) esophageal cancer diagnosed as the primary malignancy; and (IV) complete and retrievable clinical records. The exclusion criteria included patients with esophageal adenocarcinoma/adenosquamous carcinoma, any M1 disease, and incomplete medical records that affected statistical analyses. The most frequently used chemotherapy regimen was either fluorouracil plus platinum-based or docetaxel/paclitaxel-platinum-based. Courses of the chemo-regimen varied, owing to the different conditions of the patients and patient compliance. The pathologic staging system was based on the 8th edition of the Union for International Cancer Control/American Joint Committee on Cancer (UICC/AJCC). The study protocol was approved by the ethics committee of Guangdong Provincial People's Hospital (No. GDREC2019687H) and The First Affiliated Hospital of Shantou University Medical College (KY-No.2020-094). All procedures performed in this study involving human participants were in accordance with the Declaration of Helsinki (as revised in 2013). As a retrospective study, the need for informed consent was waived by both institutional review boards. A flowchart of the study design is presented in Figure 1. 


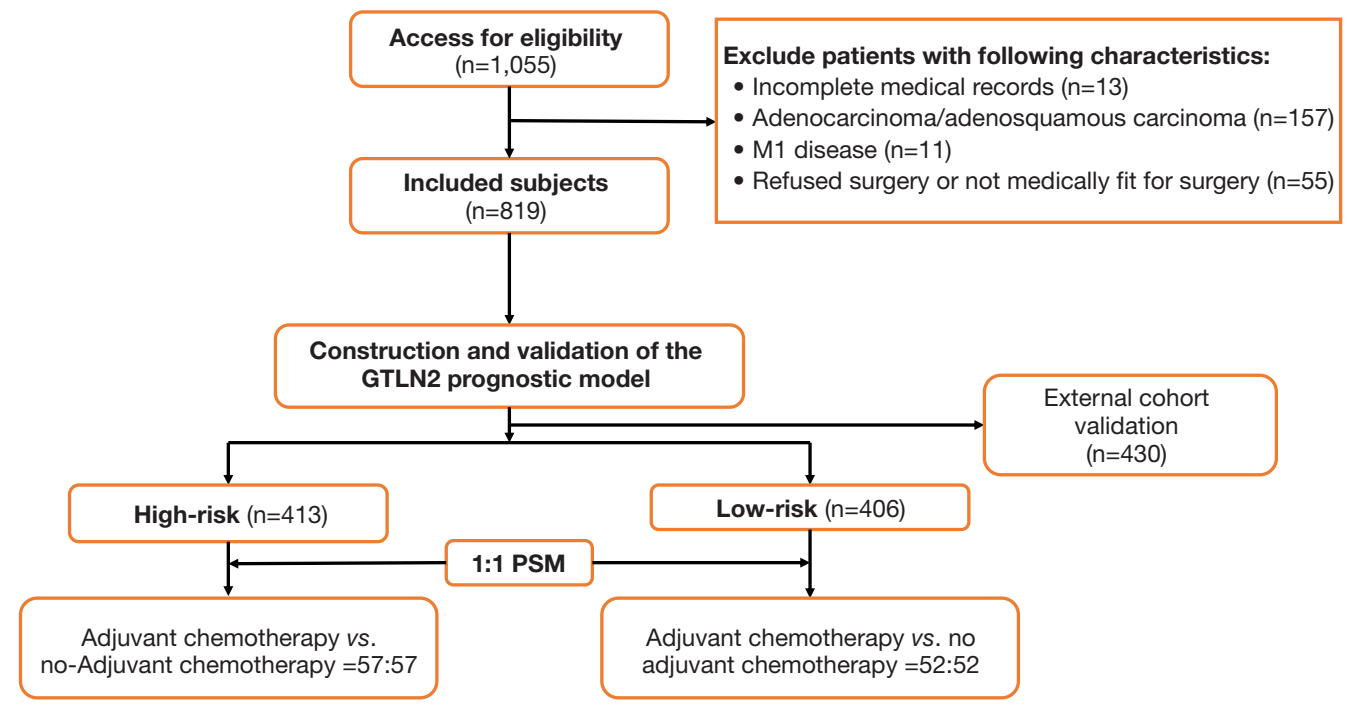

Figure 1 Flowchart of the study design. A total of 819 patients were included in the training cohort. The GTLN2 prognostic model was constructed, and patients in the training set were divided into high- and low-risk cohorts. After 1:1 propensity scoring matching, the benefit of adjuvant chemotherapy was assessed in both cohorts. The prognostic model and the benefit of adjuvant chemotherapy were validated in an external cohort. PSM, propensity score matching.

\section{Statistical analysis}

Age, as a parameter, was grouped using the median as the cutoff value. OS was considered the primary outcome of this current study and was defined as the time from diagnosis to death or last follow-up. Censored data included living or lost to follow-up individuals at the last follow-up. Intergroup comparisons of the continuous variables were analyzed using either Student's $t$-test or the Mann-Whitney test if the data did not meet the assumption of a normal distribution. Categorical variables were compared by using the chi-square test or Fisher's exact test. Univariate Cox analyses were adopted to identify survival-associated factors, whereas a multivariate Cox proportional hazards regression model was constructed to predict the independent risk factors for ESCC. We used the stepwise forward procedure in the multivariate regression analyses.

Moreover, the Kaplan-Meier method was used to calculate OS and to compare the survival differences. Thereafter, the log-rank test and Breslow test were performed to determine the statistically significant differences between survival curves. Then, we constructed a prognostic scoring system based on multivariate analyses. The formula of the risk score was presented as $\ln [\mathrm{h}(\mathrm{t}, \mathrm{X}) / \mathrm{h} 0(\mathrm{t})]=\beta 1 \times \mathrm{Z}_{\text {score }}$ prognostic factor $1+$ $\beta 2 \times Z_{\text {score }}$ prognostic factor $2+\beta 3 \times Z_{\text {score }}$ prognostic factor $3+$ $\ldots+\beta \mathrm{m} \times Z_{\text {score }}$ prognostic factorm. Each coefficient $\beta$ in the formula was standardized to ensure comparability among the contributions of each prognostic factor to the formula.

A time-dependent receiver operating characteristic (ROC) curve and the area under the curve (AUC) value were generated to evaluate the predictive power of the prognostic model. Then, we externally validated the model, and the ROC curve and AUC value of the validation cohort were generated to assess the reliability of the results. A 1:1 patient pairing was conducted using propensity score matching (PSM) to assess the potential beneficial effects of adjuvant chemotherapy in the subgroup analysis. Potential beneficiaries were defined as ESCC patients who obtained a significantly longer OS after adjuvant chemotherapy. Finally, using sensitivity analyses, we determined the robustness of the GTLN2 model. $\mathrm{P}<0.05$ (two-sided) was considered statistically significant. All statistical data were analyzed using the software "Statistical Package for Social Science" (SPSS) version 26 for Windows (SPSS Inc., Chicago, IL, USA) and R 4.0.0 (R Core Team 2020) (12). High-quality figures were generated using the $\mathrm{R}$ package.

\section{Results}

\section{Clinicopathological and survival information of the patients}

Among the 819 enrolled patients in the training cohort, the male to female ratio was approximately 4:1. Most of 
the tumors were localized in the middle portion of the esophagus. Due to a disproportionately small percentage of T4a individuals, we had to include patients staged pT1b-T3 (T1b:T2:T3 =86:216:517). In our database analysis, clinicopathological factors, including age, sex, $\mathrm{T}$ stage, $\mathrm{N}$ stage, pathological TNM (pTNM) stage, grade (G), lymphovascular invasion (LVI), perineural invasion (PNI), surgical margin, and the NCLNs, were associated with adjuvant chemotherapy. During the last follow-up, 115 patients experienced distant metastasis. In the validation cohort, the male to female ratio was 3.4:1. A total of $226(52.6 \%)$ patients received adjuvant chemotherapy. Their detailed clinicopathological information is provided in Table 1.

In the training cohort, the median OS was 64.7 (54.2-75.3) months, and the 3- and 5-year OS rates were $63.9 \% \pm 0.019$ and $52.8 \% \pm 0.021$, respectively, whereas in the validation cohort, the median OS was 69.1 (49.9-88.2) months, with 3- and 5-year survival rates of $65.2 \% \pm 0.026$ and $52.1 \% \pm 0.029$, respectively.

\section{Univariate/multivariate analysis and construction of the prognostic model in the training cobort}

Through univariate analysis, G, T, N, distant metastasis, pTNM staging, LVI, PNI, and NCLN were identified as key prognostic factors for OS. Of note, among the 9 prognosticators, NCLN was identified as a protective variable $(\mathrm{HR}=0.711 ; 95 \% \mathrm{CI}: 0.563-0.899 ; \mathrm{P}<0.017)$. Multivariate analyses revealed G, T, N, and NCLN as independent prognostic factors (Table 2).

Time-dependent covariate computation was performed to eliminate time-dependent prognostic effects from the selected variables when performing multivariate analysis and to confirm the proportional hazard assumption. To examine the subsequent interactions among these variables, we introduced the product of two independent variables into the multivariate Cox regression model. A $\mathrm{P}$ value was adopted to assess interactive effects. Eventually, the assumption of a proportional hazard was well established, and no interactions existed among the four independent prognostic factors. After computing the standardization of each coefficient $\beta$ in the formula, the risk-evaluation model was established. The formula was expressed as:

$$
\begin{aligned}
\ln \left[\frac{h(\mathrm{t}, x)}{h 0(\mathrm{t})}\right]= & 0.115 \times \text { Zscore } G+0.345 \times \text { ZscoreT }+ \\
& 0.297 \times \text { ZscoreN }-0.251 \times Z \text { scoreNCLN }
\end{aligned}
$$

A corresponding risk score was assigned to each patient in the training cohort. We named the model GTLN2. T and $\mathrm{G}$ are the abbreviations for tumor depth invasion and histological grade, respectively. Moreover, LN2 referred to two included variables that were related to lymph node (LN) characteristics. No significant differences in risk scores among various subgroups (Figure S1). Subsequently, Patients were categorized into high- and low-risk groups based on the median risk score (Figure 2). Survival analysis showed a significantly better OS (high- $v s$. low-risk group, $\mathrm{HR}=2.114 ; 95 \%$ CI: 1.699-2.629; $\mathrm{P}<0.001)$ (Figure $3 A$ ). The median survival times in the low- and high-risk groups were 104.4 and 41.8 months (95\% CI: 32.4-51.2), respectively. Subsequent multitime survival ROC analysis revealed that the 1-, 3- and 5-year AUCs were 0.692, 0.685 and 0.680 , respectively $(\mathrm{P}<0.001)$ (Figure $3 B$ ).

\section{External validation of the predictive power of the prognostic model}

We used an independent cohort from another hospital to externally validate the prognostic model. Using the formula computed from the training cohort, the risk scores were calculated and assigned to patients in the validation cohort. Survival analysis also revealed a significant difference between the high- and low-risk groups in the validation cohort (high- vs. low-risk group, HR $=1.868$; $95 \%$ CI: 1.376-2.536; $\mathrm{P}<0.001)$ (Figure $4 A$ ). The median survival time in the high-risk group was 46.0 (95\% CI: 34.68 57.32). The 1-, 3- and 5-year AUCs were $0.651,0.619$ and 0.650 , respectively $(\mathrm{P}<0.001)$ (Figure $4 B)$.

\section{Selecting beneficiaries of adjuvant chemotherapy in the training and validation cohorts}

A 1:1 patient pairing was performed using PSM to investigate the potential beneficial effects of adjuvant chemotherapy in the high- and low-risk groups. The match tolerance was set as 0.001 , after which we maximized the execution performance and randomized the case order when drawing the matches (Table S1). In total, 57 pairs of highrisk group patients were matched, and all of the covariates were comparable between the adjuvant chemotherapy and no-adjuvant chemotherapy groups. A significantly higher OS rate was found in the adjuvant chemotherapy group $\left(\chi^{2}=5.072 ; \mathrm{P}=0.024<0.05\right)$ (Figure $5 A$ ). For patients subjected to adjuvant chemotherapy, the median OS was 64.7 (95\% CI: 32.55-96.91) months, while the median 
Table 1 Baseline clinicopathological information of the study population

\begin{tabular}{|c|c|c|}
\hline Clinicopathological characteristics & Training cohort $(\mathrm{n}=819), \mathrm{n}(\%)$ & Validation cohort $(n=430), n(\%)$ \\
\hline \multicolumn{3}{|l|}{ Sex } \\
\hline Male & $657(80.2)$ & $333(77.4)$ \\
\hline Female & $162(19.8)$ & $97(22.6)$ \\
\hline \multicolumn{3}{|l|}{ Age } \\
\hline$\leq 60$ years & $443(54.1)$ & $208(48.4)$ \\
\hline$>60$ years & $376(45.9)$ & $222(51.6)$ \\
\hline \multicolumn{3}{|l|}{ Adjuvant chemotherapy } \\
\hline Yes & $318(38.8)$ & $226(52.6)$ \\
\hline Upper portion & $98(12.0)$ & $49(11.7)$ \\
\hline Middle portion & $553(67.5)$ & $253(60.2)$ \\
\hline Lower portion & $168(20.5)$ & $118(28.1)$ \\
\hline Unknown & 0 & $10(2.3)$ \\
\hline \multicolumn{3}{|l|}{ Differentiation status $(G)$} \\
\hline Well (G1) & $110(13.4)$ & $42(9.8)$ \\
\hline Mild (G2) & $550(67.2)$ & $336(78.1)$ \\
\hline Poor (G3) & $159(19.4)$ & $52(12.1)$ \\
\hline \multicolumn{3}{|l|}{ Lymph node metastases $(\mathrm{N})$} \\
\hline No & $446(54.5)$ & $261(60.7)$ \\
\hline N1 & $191(23.3)$ & $101(23.5)$ \\
\hline N2 & $109(13.3)$ & $60(14.0)$ \\
\hline N3 & $73(8.9)$ & $8(1.9)$ \\
\hline \multicolumn{3}{|l|}{ pTNM stage } \\
\hline IB & $85(10.4)$ & $36(8.4)$ \\
\hline$\| \mathrm{A}$ & $197(24.1)$ & $102(23.7)$ \\
\hline IIB & $179(21.9)$ & $132(30.7)$ \\
\hline IIIA & $47(5.7)$ & $20(4.7)$ \\
\hline IIIB & $238(29.1)$ & $132(30.7)$ \\
\hline IVA & $73(8.9)$ & $8(1.9)$ \\
\hline
\end{tabular}

Table 1 (continued) 
Table 1 (continued)

\begin{tabular}{lcc}
\hline Clinicopathological characteristics & Training cohort $(\mathrm{n}=819), \mathrm{n}(\%)$ & Validation cohort $(\mathrm{n}=430), \mathrm{n}(\%)$ \\
\hline NLCN & $17[11-23]$ & $216-28]$ \\
Median [IQR] & & $17(4.0)$ \\
LVI & $171(20.9)$ & $413(96.0)$ \\
Positive & $648(79.1)$ & Unavailable \\
Negative & & Unavailable \\
PNI & $208(25.4)$ & $611(74.6)$ \\
Positive & & $17(4.0)$ \\
Negative & $33(4.0)$ & $413(96.0)$ \\
Surgical margin & $786(96.0)$ & \\
R1 & & \\
R0 & & \\
\hline
\end{tabular}

pTNM, pathological tumor-nodal-metastasis; NLCN, number of cleared lymph node; LVI, lymphovascular invasion; PNI, perineural invasion.

Table 2 Univariate and multivariate analyses of the training cohort

\begin{tabular}{|c|c|c|c|c|c|}
\hline Variables & \multicolumn{3}{|c|}{ Univariate } & \multicolumn{2}{|l|}{ Multivariate } \\
\hline Sex & $0.798(0.605-1.053)$ & 2.561 & 0.109 & & \\
\hline Age & $1.114(0.906-1.369)$ & 1.053 & 0.305 & & \\
\hline Tumor location & & 1.772 & 0.412 & & \\
\hline Middle portion & $0.822(0.593-1.139)$ & & & & \\
\hline Lower portion & $0.779(0.531-1.143)$ & & & & \\
\hline Histological grade (G) & $1.307(1.0888-1.572)$ & 8.147 & $0.015^{\star}$ & $1.223(1.013-1.477)$ & $0.036^{*}$ \\
\hline Tumor invasion depth $(T)$ & $1.740(1.431-2.116)$ & 32.070 & $<0.001^{*}$ & $1.662(1.362-2.029)$ & $<0.001^{*}$ \\
\hline pTNM stage & $1.292(1.205-1.384)$ & 71.862 & $<0.001^{*}$ & & \\
\hline $\mathrm{NLCN}$ & $0.711(0.563-0.899)$ & 8.103 & $0.017^{*}$ & $0.962(0.962-0.986)$ & $<0.001^{*}$ \\
\hline LVI & 2.159 (1.721-2.709) & 46.447 & $<0.001^{*}$ & & \\
\hline PNI & $1.614(1.289-2.021)$ & 17.775 & $<0.001^{*}$ & & \\
\hline Surgical margin & $1.302(0.763-2.225)$ & 0.941 & 0.332 & & \\
\hline
\end{tabular}

*, $\mathrm{P}<0.05$. pTNM, pathological tumor-nodal-metastasis; NLCN, number of cleared lymph node; LVI, lymphovascular invasion; PNI, perineural invasion. 

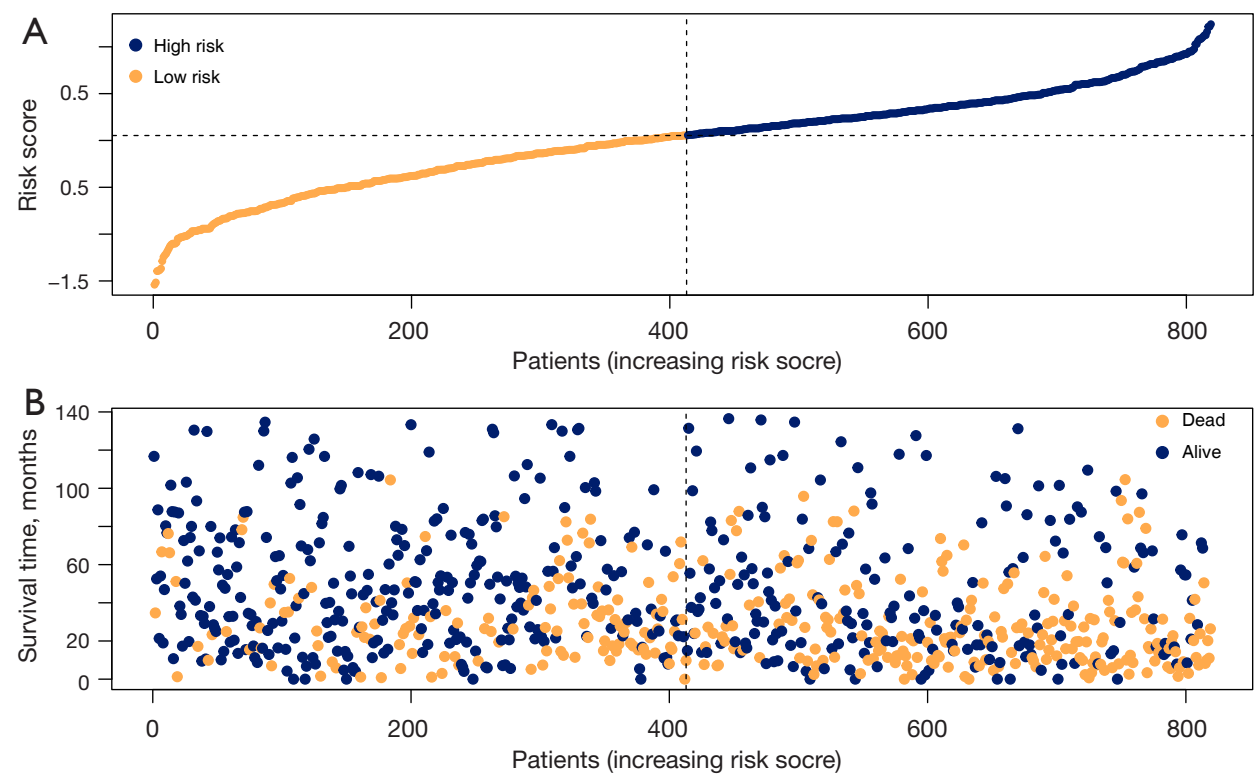

Figure 2 Risk score model plot and survival plot. (A) Risk score model of the training set. The X-axis represents patient numbers that were ranked from low to high by their risk score. The $\mathrm{Y}$-axis indicates the risk score. (B) Survival plot of the training set. The $\mathrm{X}$-axis represents the patient numbers ranked from low to high by their risk score. The Y-axis indicates the survival time.

A

A

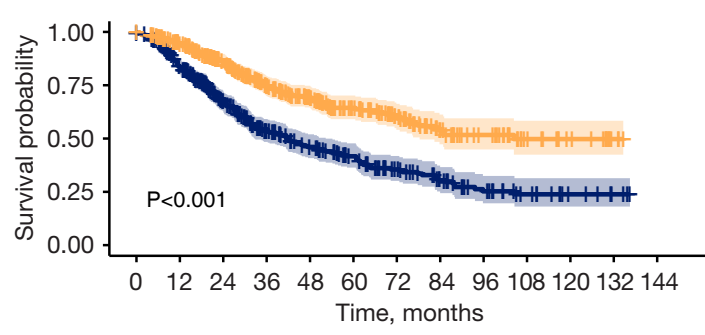

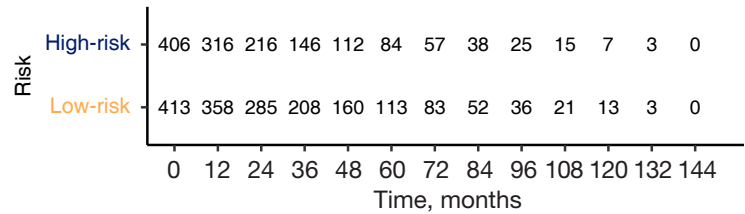

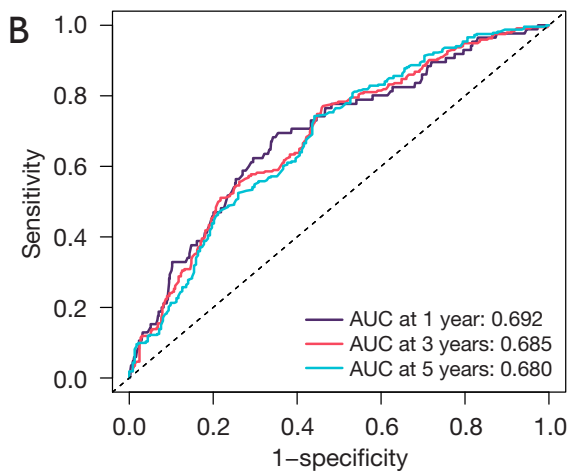

Figure 3 Survival differences in stratified risk groups and internal validation of the GTLN2 model in the training set. (A) Survival difference between high- and low-risk groups in the training set. Significantly better OS benefits were observed in the low-risk group than in the highrisk group in the training cohort. (B) Multitime survival ROC curve of the GTLN2 model in the training set. OS, overall survival; ROC, receiver operating characteristic; AUC, area under the curve.

OS in the non-adjuvant chemotherapy group was 32.6 (95\% CI: 20.37-44.89) months. The analysis of high-risk patients in the validation cohort also showed better OS in the postoperative chemotherapy treatment group than in the observation group $\left(\chi^{2}=4.853 ; \mathrm{P}=0.062\right)$ (Figure $5 B$ ). In contrast, the OS curves demonstrated a trend toward deterioration in the adjuvant chemotherapy group in patients with a lower risk $\left(\chi^{2}=2.415 ; \mathrm{P}=0.28\right)$ (Figure 5C). 

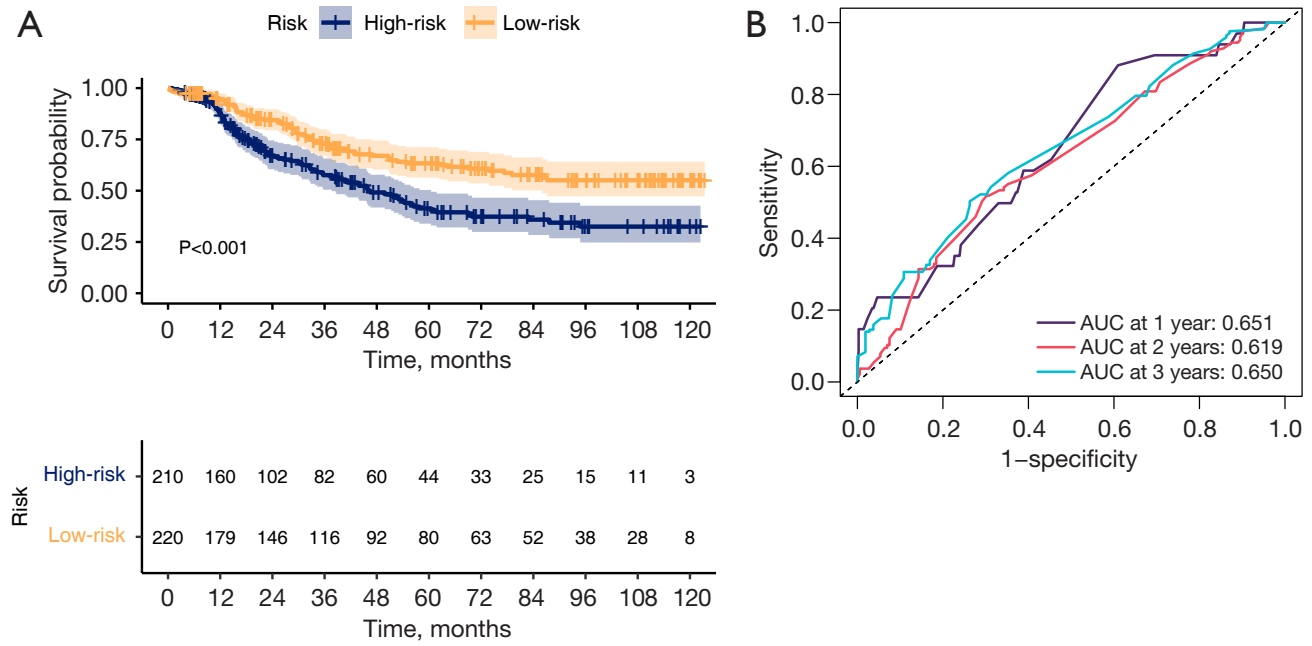

Figure 4 Survival differences in stratified risk groups and internal validation of the GTLN2 model in the validation set. (A) Survival difference between high- and low-risk groups in the validation set. Significantly better OS benefits were observed in the low-risk group than in the high-risk group. (B) Multitime survival ROC curve of the GTLN2 model in the validation set. OS, overall survival; ROC, receiver operating characteristic; AUC, area under the curve.

Similarly, the OS in the lower-risk validation group did not improve $\left(\chi^{2}=2.386 ; \mathrm{P}=0.28\right.$ ) (Figure $\left.5 D\right)$.

\section{Sensitivity analysis}

By introducing and eliminating different variables significantly associated with OS in the univariate analyses, we constructed 8 regression models and compared them. We performed a series of analyses confirming the proportional hazard assumption, interactions among the variables, and the ROC analyses. Of the 8 prognostic models, 2 did not meet the proportionality assumption tests for Cox regression, and the GTLN2 model exhibited the highest AUC values compared to the others.

\section{Discussion}

The overall prognosis of ESCC is poor. To date, the TNM staging system is the most widely used tool for predicting patient outcomes. However, significant survival differences among patients with the same pathological stage have been observed. Hence, a more accurate, effective prognostic model is urgently needed. Moreover, the therapeutic efficacy of adjuvant chemotherapy has been the subject of intense debate.

Several assessments have reported encouraging survival benefits of adjuvant chemotherapy (4-6). However, no significant association of prolonged OS with chemotherapy initiation after esophagectomy surgery has been described (7). In addition to these controversial findings, a lack of high-level evidence, such as phase III randomized controlled trials, hinders a wider range of clinical applications of adjuvant chemotherapy (8). Moreover, due to the tumor heterogeneity of esophageal cancer patients (13) and other unknown reasons, treatment effects vary. Notably, tumor heterogeneity could explain these controversial conclusions; however, further research is required to elucidate such a theory.

Based on the current understanding, we proposed a novel and reliable method for accurately selecting adjuvant chemotherapy beneficiaries via a validated multivariate prognostic model (the GTLN2 model). By stratifying patients into various risk groups based on this model, we revealed the survival differences of ESCC patients and identified a more suitable subgroup to receive adjuvant chemotherapy.

Consistent with the findings of Rice et al. $(14,15)$ and other clinical findings, the tumor differentiation status $(\mathrm{G})$ of ESCC markedly affects survival $(9,16)$. In our study, we identified $\mathrm{G}$ as one of the independent prognostic factors, along with $\mathrm{T}, \mathrm{N}$, and NCLN. Apart from the well-established prediction power of T, N, and G, we found that the number of LNs at clearance significantly contributed to the OS and provided insights into devising 

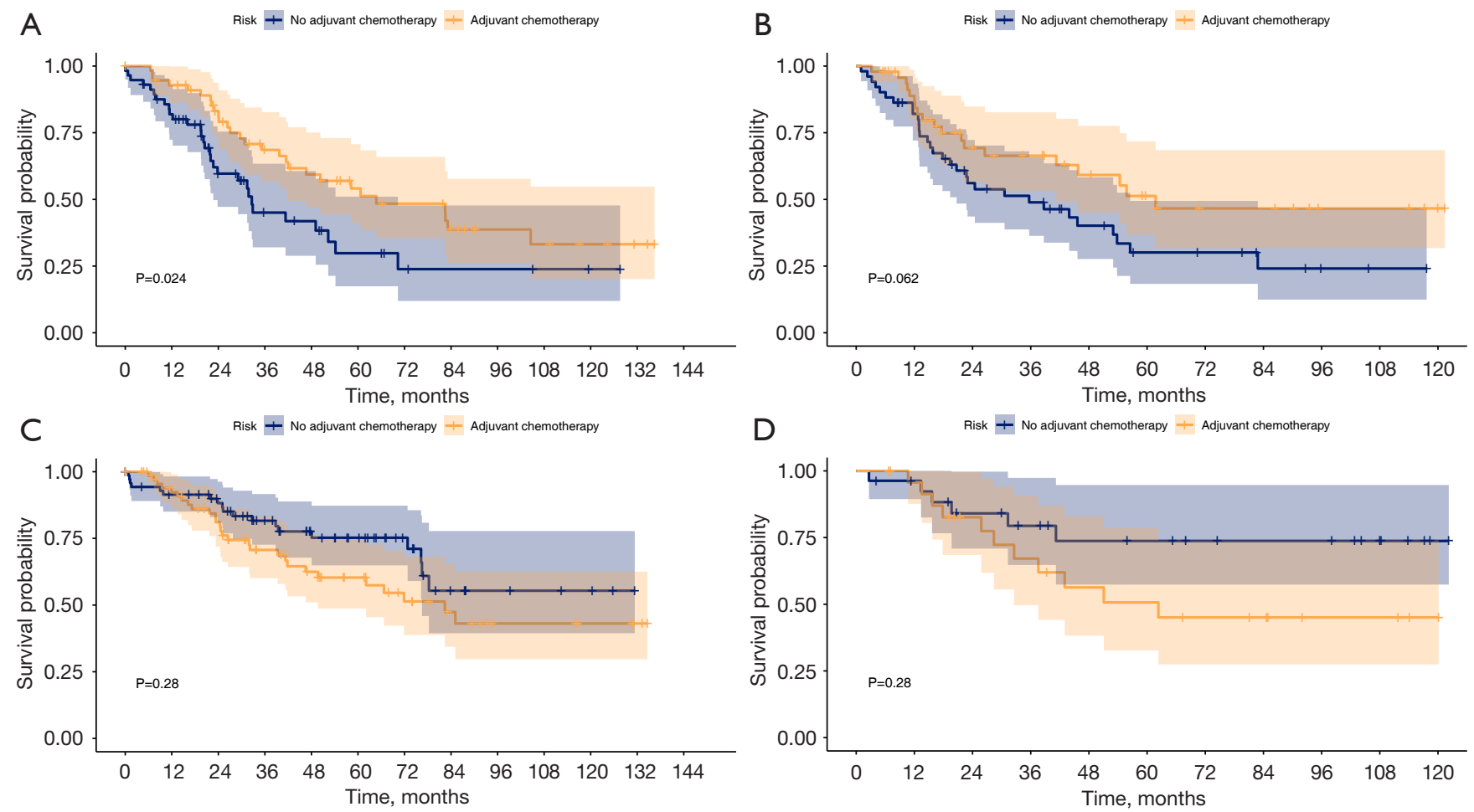

Figure 5 Survival difference between the adjuvant chemotherapy group and the no-adjuvant chemotherapy group in both the training and validation sets. (A) Analysis of the beneficial effects of adjuvant chemotherapy in high-risk patients in the training cohort. Significantly better OS benefits were observed in the adjuvant chemotherapy treatment group than in the no-adjuvant chemotherapy group in the highrisk training cohort. (B) Analysis of the beneficial effects of adjuvant chemotherapy in high-risk patients in the validation cohort. Better OS benefits were observed in the adjuvant chemotherapy treatment group than in the no-adjuvant chemotherapy group in the high-risk validation cohort. (C) Analysis of the beneficial effects of adjuvant chemotherapy in low-risk patients in the training set. No survival benefits of adjuvant chemotherapy treatment were observed in the training set. (D) Analysis of the beneficial effects of adjuvant chemotherapy in low-risk patients in the validation set. No survival benefits of the low-risk group with adjuvant chemotherapy treatment were observed in the validation set. OS, overall survival.

treatment strategies. Elsewhere, Li et al. [2019] reported that integrating NCLNs with the positive lymph node ratio (PLNR) could predict the outcome of ESCC patients undergoing adjuvant chemotherapy (17). Consistent with our findings, Samson et al. suggested NCLN as a protective prognosticator for esophagectomized ESCC patients (18).

By integrating these validated independent prognosticators, we developed a multivariate prognostic model and categorized ESCC patients into high- and lowrisk groups. Using Kaplan-Meier survival analysis, we revealed significant differences in OS (high- vs. low-risk group, $\mathrm{HR}=2.114 ;$ 95\% CI: $1.699-2.629 ; \mathrm{P}<0.001)$ in favor of patients with lower risk scores. Sequential prediction power computation and external cohort validation confirmed the robustness of the model.
Multiple studies have made attempts to develop effective prognostic models by integrating clinicopathological factors of potential prognostic significance to guide therapeutic intervention and tertiary prevention in esophagectomized ESCC patients (19-24). In this way, they aim to offer survival benefits and improve the OS rate. However, a limited number of published studies have examined the value of a prognostic scoring system for predicting the effects of adjuvant chemotherapy. To advance postoperative therapeutic decision-making, Ma and colleagues presented a novel prognostic model that included multiple clinical variables for node-negative ESCC patients (24). Other studies by Su et al. (19) and Deng et al. [2019] proposed the use of nomograms in the successful prediction of the OS of ESCC patients and to assist in decision-making during 
adjuvant chemotherapy (21).

Although shared similarities exist, several aspects of our study differ from the abovementioned previous reports. First, we included surgically fit cT1b-3 patients, whereas other studies only focused on specific smaller groups of patients $(21,24)$, which limits the applicability of their prognostic model. Second, instead of applying the existing prognostic scoring systems $(23,25)$, we proposed a novel prognostic model integrated with universally recognized independent prognosticators. The prognostic model was generated after factor interaction analysis and time-dependent covariate computation. This was conducted to minimize the potential bias for nonproportionality and over/underestimating the predictive power of certain prognostic factors. Furthermore, external cohort validation undoubtedly highlighted the applicability and utility of our work.

More importantly, the subgroup analysis affirmed that our prognostic model could aid in predicting the effects of adjuvant chemotherapy in different patient groups. Based on our prognostic model, we separately analyzed the potential benefits of adjuvant chemotherapy in the high- and low-risk groups. Notably, in the high-risk group, adjuvant chemotherapy improved OS $\left(\chi^{2}=5.072\right.$; $\mathrm{P}=0.024<0.05)$. On the other hand, no difference in OS was found between the treatment and the nontreatment groups in lower-risk patients. These results demonstrated that adjuvant chemotherapy is beneficial for only highly selected patient groups. Moreover, from our perspective, adjuvant chemotherapy will likely do more harm than good to patients in the lower-risk groups.

By stratifying patients based on their distinctive characteristics as well as shared similarities, we identified ESCC patients who are likely to benefit from chemotherapy. Previously, a prognostic scoring model was proposed for the utility of induction chemotherapy (9). Furthermore, novel scoring systems have been developed to predict postoperative chemotherapeutic benefits in other cancer types, such as pancreatic cancer (26). However, to date, no report has been published describing a validated scoring system for screening ESCC patient groups.

In addition, there were some limitations and suggested improvements to the current study. First, the retrospective nature of this study could introduce potential selection biases despite adopting strict selection and exclusion criteria. Future large-scale, prospective studies are required to confirm the results. Second, the application of the GTLN2 model was limited to only Chinese T1b-3 ESCC patients, and extended analyses involving patients of other ethnicities and broader $\mathrm{T}$ spectra should be considered. Finally, although we developed and validated the utility and applicability of the prognostic scoring system for screening beneficiaries of adjuvant chemotherapy, additional research is required to compare and select a more effective chemotherapeutic regimen.

In conclusion, with the aid of a novel and validated prognostic scoring model, we stratified high- and lowrisk groups of esophagectomized ESCC patients with significant survival differences. Subgroup analysis indicated that the high-risk cohort was the preferred candidate for adjuvant chemotherapy, while the low-risk cohort was not. These findings could provide supplementary evidence for oncologists when considering whether to initiate early adjuvant chemotherapy. However, multicenter, prospective research is required to validate these findings.

\section{Acknowledgments}

We are grateful to the Freescience Editing Service for English language editing.

Funding: This work was supported by a grant from the 2020-2021 Popularization of Science and Technology Innovation Special Project of Guangdong Province of China (No. 2020A1414070007), and the Science and Technology Program of Guangzhou, China (201704020107).

\section{Footnote}

Reporting Checklist: The authors have completed the STROBE reporting checklist. Available at https://atm. amegroups.com/article/view/10.21037/atm-22-46/rc

Data Sharing Statement: Available at https://atm.amegroups. com/article/view/10.21037/atm-22-46/dss

Conflicts of Interest: All authors have completed the ICMJE uniform disclosure form (available at https://atm. amegroups.com/article/view/10.21037/atm-22-46/coif). All authors report that this work was supported by a grant from the 2020-2021 Popularization of Science and Technology Innovation Special Project of Guangdong Province of China (No. 2020A1414070007), and the Science and Technology Program of Guangzhou, China (201704020107). The authors have no other conflicts of interest to declare.

Ethical Statement: The authors are accountable for all aspects of the work in ensuring that questions related 
to the accuracy or integrity of any part of the work are appropriately investigated and resolved. All procedures performed in this study involving human participants were in accordance with the Declaration of Helsinki (as revised in 2013). The study protocol was approved by the ethics committee of Guangdong Provincial People's Hospital (No. GDREC2019687H) and The First Affiliated Hospital of Shantou University Medical College (KY-No.2020-094). As a retrospective study, the need for informed consent was waived by both institutional review boards.

Open Access Statement: This is an Open Access article distributed in accordance with the Creative Commons Attribution-NonCommercial-NoDerivs 4.0 International License (CC BY-NC-ND 4.0), which permits the noncommercial replication and distribution of the article with the strict proviso that no changes or edits are made and the original work is properly cited (including links to both the formal publication through the relevant DOI and the license). See: https://creativecommons.org/licenses/by-nc-nd/4.0/.

\section{References}

1. Allemani C, Matsuda T, Di Carlo V, et al. Global surveillance of trends in cancer survival 2000-14 (CONCORD-3): analysis of individual records for 37513025 patients diagnosed with one of 18 cancers from 322 population-based registries in 71 countries. Lancet 2018;391:1023-75.

2. Lin $\mathrm{Y}$, Totsuka $\mathrm{Y}, \mathrm{He} \mathrm{Y}$, et al. Epidemiology of esophageal cancer in Japan and China. J Epidemiol 2013;23:233-42.

3. National Comprehensive Cancer Network. Esophageal and Esophagogastric Junction Cancers (Version 3.2021). Available online: https://www.nccn.org/professionals/ physician_gls/pdf/esophageal.pdf (Accessed June 22, 2021).

4. Qin RQ, Wen YS, Wang WP, et al. The role of postoperative adjuvant chemotherapy for lymph nodepositive esophageal squamous cell carcinoma: a propensity score matching analysis. Med Oncol 2016;33:31.

5. Wong AT, Shao M, Rineer J, et al. The Impact of Adjuvant Postoperative Radiation Therapy and Chemotherapy on Survival After Esophagectomy for Esophageal Carcinoma. Ann Surg 2017;265:1146-51.

6. Zhao P, Yan W, Fu H, et al. Efficacy of postoperative adjuvant chemotherapy for esophageal squamous cell carcinoma: A meta-analysis. Thorac Cancer 2018;9:1048-55.

7. Pasquer A, Gronnier C, Renaud F, et al. Impact of Adjuvant Chemotherapy on Patients with Lymph Node-
Positive Esophageal Cancer who are primarily Treated with Surgery. Ann Surg Oncol 2015;22 Suppl 3:S1340-9.

8. National Comprehensive Cancer Network. Esophageal and Esophagogastric Junction Cancers (Version 2.2020). 2020. Available online: https://www.ncen.org/ professionals/physician_gls/pdf/esophageal.pdf (Accessed July 7, 2020).

9. Xi M, Liao Z, Deng W, et al. A Prognostic Scoring Model for the Utility of Induction Chemotherapy Prior to Neoadjuvant Chemoradiotherapy in Esophageal Cancer. J Thorac Oncol 2017;12:1001-10.

10. Nomura M, Shitara K, Kodaira T, et al. Prognostic impact of the 6th and 7th American Joint Committee on Cancer TNM staging systems on esophageal cancer patients treated with chemoradiotherapy. Int J Radiat Oncol Biol Phys 2012;82:946-52.

11. Duan J, Deng T, Ying G, et al. Prognostic nomogram for previously untreated patients with esophageal squamous cell carcinoma after esophagectomy followed by adjuvant chemotherapy. Jpn J Clin Oncol 2016;46:336-43.

12. R Core Team (2020). R: A language and environment for statistical computing. Vienna: R Foundation for Statistical Computing, 2020.

13. Yan T, Cui H, Zhou Y, et al. Multi-region sequencing unveils novel actionable targets and spatial heterogeneity in esophageal squamous cell carcinoma. Nat Commun 2019;10:1670.

14. Rice TW, Patil DT, Blackstone EH. 8th edition AJCC/UICC staging of cancers of the esophagus and esophagogastric junction: application to clinical practice. Ann Cardiothorac Surg 2017;6:119-30.

15. Rice TW, Ishwaran H, Ferguson MK, et al. Cancer of the Esophagus and Esophagogastric Junction: An Eighth Edition Staging Primer. J Thorac Oncol 2017;12:36-42.

16. Ajani JA, Correa AM, Hofstetter WL, et al. Clinical parameters model for predicting pathologic complete response following preoperative chemoradiation in patients with esophageal cancer. Ann Oncol 2012;23:2638-42.

17. Li Y, Zhao W, Ni J, et al. Predicting the Value of Adjuvant Therapy in Esophageal Squamous Cell Carcinoma by Combining the Total Number of Examined Lymph Nodes with the Positive Lymph Node Ratio. Ann Surg Oncol 2019;26:2367-74.

18. Samson P, Puri V, Broderick S, et al. Extent of Lymphadenectomy Is Associated With Improved Overall Survival After Esophagectomy With or Without Induction Therapy. Ann Thorac Surg 2017;103:406-15. 
19. Su D, Zhou X, Chen Q, et al. Prognostic Nomogram for Thoracic Esophageal Squamous Cell Carcinoma after Radical Esophagectomy. PLoS One 2015;10:e0124437.

20. Zheng Y, Fu S, He T, et al. Predicting prognosis in resected esophageal squamous cell carcinoma using a clinical nomogram and recursive partitioning analysis. Eur J Surg Oncol 2018;44:1199-204.

21. Deng $W$, Zhang $W$, Yang J, et al. Nomogram to Predict Overall Survival for Thoracic Esophageal Squamous Cell Carcinoma Patients After Radical Esophagectomy. Ann Surg Oncol 2019;26:2890-8.

22. Lee L, Ronellenfitsch U, Hofstetter WL, et al. Predicting lymph node metastases in early esophageal adenocarcinoma using a simple scoring system. J Am Coll Surg 2013;217:191-9.

23. Vashist YK, Loos J, Dedow J, et al. Glasgow Prognostic Score is a predictor of perioperative and long-term

Cite this article as: Zhou Z, Huang S, Ben X, Zhuang W, Hong L, Xie Z, Zhang D, Xie L, Zhou H, Tang J, Chen G, Wu H, Qiao G. A novel prognostic model: which group of esophageal squamous cell carcinoma patients could benefit from adjuvant chemotherapy. Ann Transl Med 2022;10(4):182. doi: 10.21037/atm-22-46 outcome in patients with only surgically treated esophageal cancer. Ann Surg Oncol 2011;18:1130-8.

24. Ma G, Zhang X, Ma Q, et al. A novel multivariate scoring system for determining the prognosis of lymph nodenegative esophageal squamous cell carcinoma following surgical therapy: an observational study. Eur J Surg Oncol 2015;41:541-7.

25. Takeda FR, Tustumi F, de Almeida Obregon C, et al. Prognostic Value of Tumor Regression Grade Based on Ryan Score in Squamous Cell Carcinoma and Adenocarcinoma of Esophagus. Ann Surg Oncol 2020;27:1241-7.

26. Liu L, Xu HX, He M, et al. A novel scoring system predicts postsurgical survival and adjuvant chemotherapeutic benefits in patients with pancreatic adenocarcinoma: Implications for AJCC-TNM staging. Surgery 2018;163:1280-94. 

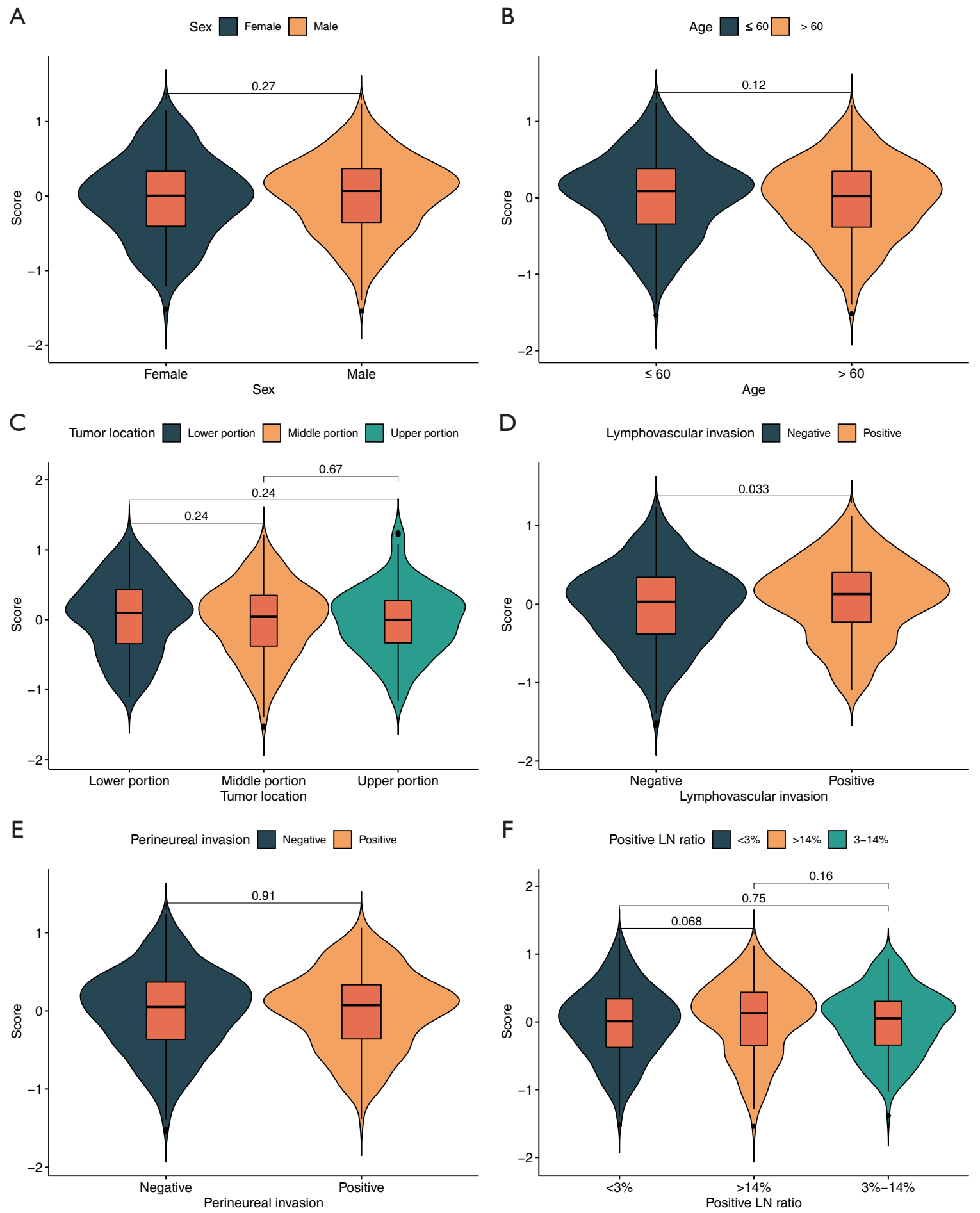

Figure S1 Differences in risk score among various subgroups. (A) Sex. No significant difference between males and females. (B) Age. No significant difference between age $>60$ and $\leq 60$. (C) Tumor location. There were no significant differences among the lower, middle and upper portions. (D) LVI. Significant differences were observed between the negative LVI group and the positive LVI group. (E) PNI. There was no significant difference between the negative PNI group and the positive PNI group. (F) PLNR. There were no significant differences among the different PLNR groups. LVI, lymphovascular invasion; PNI, perineural invasion; PLNR, positive lymph node ratio; LN, lymph node. 
Table S1 Clinicopathological factors of the study population before and after PSM in high-risk groups

\begin{tabular}{|c|c|c|c|c|c|c|}
\hline \multirow[b]{2}{*}{ Variables } & \multicolumn{3}{|c|}{ High-risk cohort } & \multicolumn{3}{|c|}{ PSM high-risk cohort } \\
\hline & $\begin{array}{c}\text { Non-adjuvant chemo, } \\
\mathrm{n}(\%)\end{array}$ & $\begin{array}{c}\text { Adjuvant chemo, } \\
\mathrm{n}(\%)\end{array}$ & $P$ value & $\begin{array}{c}\text { Non-adjuvant chemo, } \\
\mathrm{n}(\%)\end{array}$ & $\begin{array}{c}\text { Adjuvant chemo, } \\
\mathrm{n}(\%)\end{array}$ & $P$ value \\
\hline Sex & & & 0.004 & & & 0.542 \\
\hline Male & $150(76.1)$ & $182(87.1)$ & & $52(91.2)$ & $50(87.7)$ & \\
\hline Female & 47 (23.9) & 27 (12.9) & & $5(8.8)$ & $7(12.23)$ & \\
\hline Age & & & & & & 0.442 \\
\hline$\leq 60$ years & $86(43.7)$ & $133(63.6)$ & $<0.001$ & $33(57.9)$ & $37(64.9)$ & \\
\hline$>60$ years & $111(56.3)$ & $76(36.4)$ & & $24(42.1)$ & $20(35.1)$ & \\
\hline Neoadjuvant chemo & & & 0.288 & & & 0.793 \\
\hline Yes & $29(14.7)$ & $39(18.7)$ & & $8(14.0)$ & $48(84.2)$ & \\
\hline No & $168(85.3)$ & $170(81.3)$ & & $49(86.0)$ & $9(15.8)$ & \\
\hline Tumor location & & & 0.64 & & & 0.075 \\
\hline Upper portion & $18(9.1)$ & $25(10.6)$ & & $3(5.3)$ & $8(14.0)$ & \\
\hline Middle portion & $131(66.5)$ & $133(63.6)$ & & $43(75.4)$ & $32(56.1)$ & \\
\hline Lower portion & $48(24.4)$ & $51(24.4)$ & & $11(19.3)$ & $17(29.8)$ & \\
\hline Differentiation status $(G)$ & & & 0.724 & & & 0.805 \\
\hline Well (G1) & $14(7.1)$ & $15(7.2)$ & & $6(10.5)$ & $6(10.5)$ & \\
\hline Mild (G2) & $136(69.0)$ & $137(65.6)$ & & $35(61.4)$ & $38(66.7)$ & \\
\hline Poor (G3) & $47(23.9)$ & $57(27.3)$ & & $16(28.1)$ & $13(22.8)$ & \\
\hline Tumor depth (T) & & & 0.229 & & & 1 \\
\hline $\mathrm{T} 1 \mathrm{~b}$ & $1(0.5)$ & $0(0.0)$ & & $0(0.0)$ & $0(0.0)$ & \\
\hline $\mathrm{T} 2$ & $17(8.6)$ & 27 (12.9) & & $6(10.5)$ & $6(10.5)$ & \\
\hline T3 & $179(90.9)$ & $182(87.1)$ & & $51(89.5)$ & $51(89.5)$ & \\
\hline Lymph node metastasis (N) & & & $<0.001$ & & & 0.932 \\
\hline No & $85(43.1)$ & $40(19.1)$ & & $17(29.8)$ & 19 (33.3) & \\
\hline $\mathrm{N} 1$ & $58(29.4)$ & $65(31.1)$ & & $19(33.3)$ & $18(31.6)$ & \\
\hline N2 & $27(13.7)$ & $64(30.6)$ & & $14(24.6)$ & $13(22.8)$ & \\
\hline N3 & $27(13.7)$ & $40(19.1)$ & & $7(12.3)$ & $7(12.3)$ & \\
\hline pTNM & & & $<0.001$ & & & 0.932 \\
\hline$\| A$ & $22(11.2)$ & $9(4.3)$ & & $3(5.3)$ & $5(8.8)$ & \\
\hline IIB & $63(32.0)$ & $31(14.8)$ & & $14(24.6)$ & $14(24.6)$ & \\
\hline IIIA & $6(3.0)$ & $4(1.9)$ & & $2(3.5)$ & $1(1.8)$ & \\
\hline IIIB & $79(40.1)$ & $125(59.8)$ & & $31(54.4)$ & $30(52.6)$ & \\
\hline IVA & $27(13.7)$ & $40(19.1)$ & & $7(12.3)$ & 7 (12.3) & \\
\hline cTNM & & & $<0.001$ & & & 0.979 \\
\hline II & $91(46.2)$ & 44 (21.1) & & 19 (33.3) & $20(35.1)$ & \\
\hline III & $79(40.1)$ & $125(59.8)$ & & $31(54.4)$ & $30(52.6)$ & \\
\hline IVA & $27(13.7)$ & $40(19.1)$ & & 7 (12.3) & 7 (12.3) & \\
\hline LVI & & & 0.013 & & & 0.691 \\
\hline Negative & $50(25.4)$ & 132 (63.2) & & 37 (64.9) & $39(68.4)$ & \\
\hline Positive & 147 (74.6) & 77 (36.8) & & $20(35.1)$ & 18 (31.6) & \\
\hline $\mathrm{PNI}$ & & & 0.079 & & & 0.845 \\
\hline Negative & 137 (69.5) & $128(61.2)$ & & $37(64.9)$ & $36(63.2)$ & \\
\hline Positive & $60(30.5)$ & $81(38.8)$ & & $20(35.1)$ & $21(36.8)$ & \\
\hline Surgical margin & & & 0.13 & & & 0.647 \\
\hline Ro & $191(97.0)$ & 196 (93.8) & & 55 (96.5) & $54(94.7)$ & \\
\hline $\mathrm{R} 1$ & $6(3.0)$ & $13(6.2)$ & & $2(3.5)$ & $3(5.3)$ & \\
\hline
\end{tabular}

PSM, propensity score matching; pTNM, pathological tumor-nodal-metastasis; cTNM, clinical tumor-nodal-metastasis; LVI, lymphovascular invasion; $\mathrm{PNI}$, perineural invasion. 